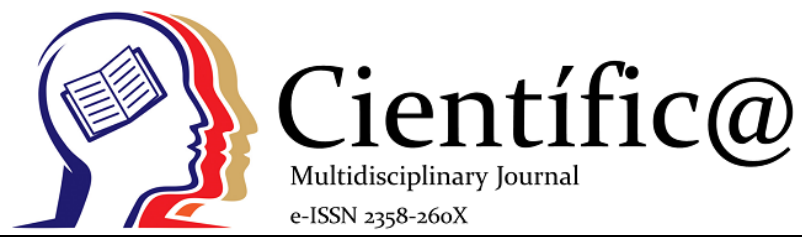

\title{
ANÁLISE MULTIVARIADA EM CARACTERÍSTICAS VEGETATIVAS E REPRODUTIVAS EM PROGÊNIES DE AÇAIZEIROS
}

\author{
MULTIVARIAL ANALYSIS IN PLANT AND REPRODUCTIVE CHARACTERISTICS IN \\ PROGENIES OF AÇAIZEIROS
}

\author{
Gilberto Ken Iti Yokomizo ${ }^{1 *}$; João Tomé de Farias Neto ${ }^{2}$; Kuang Hongyu ${ }^{3}$; Francisco de \\ Oliveira Cruz Júnior ${ }^{4}$
}

\footnotetext{
${ }^{1}$ Embrapa Amapá - * gilberto.yokomizo@embrapa.br

${ }^{2}$ Embrapa Amazônia Oriental

${ }^{3}$ Universdade Federal do Mato Grosso

${ }^{4}$ Instituto de Pesquisa Científicas e Tecnológicas do Amapá
}

\section{Info}

Recebido: 05/2019

Publicado: $10 / 2020$

DOI: $10.29247 / 2358-260 X .2020 v 7 i 1.4529$

ISSN: 2358-260X

\section{Palavras-Chave}

Euterpe oleracea; dendrograma; biplot; melhoramento genético

\section{Keywords:}

Euterpe oleracea; dendrogram; biplot; genetic breeding

\section{Resumo}

0 fruto do açaizeiro tem seu consumo em expansão no Brasil e no exterior, utilizando-se descritores em quantidade que dificulta a avaliação no campo e a seleção, sendo que a análise multivariada facilitaria este processo, portanto o objetivo com este trabalho foi analisar o comportamento das características visando determinar quais apresentam maior contribuição nas manifestações fenotípicas e também aquelas com maior potencial a serem utilizados para seleção. 0 delineamento experimental adotado foi de blocos ao acaso com duas repetições, cinco plantas por parcela e 175 progênies. Foram avaliadas as características: Lançamento Foliar (FLF); Folhas Maduras Abertas (FAB); Folhas Senescentes (FSE); Número de Facões ou Espatas Emitidas (FC); Cacho com Inflorescência em Floração (FL); Formação de Cacho Verde (FV); Cachos com Formação de Frutos Verdes (FF); Cacho com Frutos Maduros (FM); Tamanho do Cacho (TM); número de perfilhos presentes na estipe (NE); número cachos sem frutos (VS). As conclusões obtidas foram de que a análise inicial indica o uso de duas características para seleção (FAB e outra dentre as demais); ao ampliar o número de características pelo dendrograma deve utilizar FAB, escolher NE ou TM e optar por uma entre as demais; o Biplot refinou a escolha das caraterísticas, permitindo selecionar FAB, TM, FV e FF, com contribuições positivas; o uso de metodologias combinadas, principalmente com o Biplot permite corrigir erros de 


\section{INTRODUÇÃO}

O açaizeiro é uma palmeira da família Arecaceae, vastamente disseminada e cultivada na Amazônia brasileira. Ocorre de forma natural na região amazônica e possui grande importância econômica para a fruticultura regional, principalmente para o Pará cujas produção, comercialização e consumo de polpa movimentam grandes mercados (Neves et al., 2015), sendo o suco de açaí um produto ícone deste Estado, representando o principal produto extraído da palmeira (Silvestre et al., 2016).

Com a popularização do apelo ecológico e das descobertas das qualidades nutricionais a comercialização da polpa de açaí tem crescido gradativamente juntamente com uma expansão para outros mercados nacionais e atingindo até níveis internacionais (Pagliarussi, 2010). Esta produção torna o Brasil o maior produtor, consumidor e exportador de polpa de açaí (Menezes, 2005), produzindo em 2018 a quantidade de 1.510 .022 t, numa área de 198.497 ha, gerando cerca de $\mathrm{R} \$ 3,265$ bilhões, com a região Norte representando $99 \%$ destes valores, destacando-se o Pará com mais de 95\% do total brasileiro (IBGE, 2020). No Estado do Pará o açaí envolve mais de 13.000 produtores, tendo grande importância socioeconômica no estado, abrangendo 300 mil pessoas de 54 municípios. (COMEX DO BRASIL, 2017). Na capital, Belém, estima-se que 25 mil pessoas atuam de forma direta e indireta, na comercialização do açaí, como extração, transporte, comercialização e industrialização, e com isso correspondendo a $70 \%$ da fonte de renda da população ribeirinha (Nogueira et al., 2013; Rodrigues et al., 2015a; 2015b).

A Secretaria de Desenvolvimento Agropecuário e da Pesca (SEDAP), avalia que 60\% da produção do açaí é consumido internamente no Pará, 30\% em outros estados do Brasil e 10\% são exportados para outros países, revelando o mercado interno e nacional como grandes consumidores do fruto e o mercado externo com potencial apreciável de crescimento (Pessoa e Teixeira, 2012; Tavares e Homma, 2015). Na exportação, destacam-se alguns países da Europa, nos Estados Unidos, Japão e China, (Schreckinger et al., 2010), adicionalmente também sendo exportado para Holanda e Coréia do Sul (Carvalho et al., 2017), impulsionado em função de suas propriedades antioxidantes, da composição nutricional, rica em fibras, lipídeos, fenóis e a antocianina que podem estar relacionados à prevenção de doenças cardiovasculares (Moura Rocha, 2015; Yamaguchi et al., 2015). Sendo rico também em proteínas, vitamina $\mathrm{E}$, e minerais, como manganês, cobre, boro e cromo (Silva et al. 2017) e apresentar efeito hipocolesterolêmico (Souza et al., 2011).

Com a valorização e o acréscimo na demanda dos diversos mercados pelo açaí, há marcante necessidade de aumento do quantitativo de frutos necessários para atender aos consumidores. Neste contexto, está ocorrendo expansão dos açaizais manejados, em áreas de várzeas e em áreas de terra firme (Farias Neto et al., 2011). Observando-se também na mesorregião do Pará, Nordeste Paraense, a incorporação da tecnologia da irrigação no plantio de açaizeiros no período de estiagem, visando permitir uma produção de açaí na entressafra (Nogueira et al., 2013). Uma parceria entre instituições de pesquisa e a Secretaria de Agricultura do Pará (SEDAP) visa expandir a área de produção em 50 mil ha até 2024, permitindo um incremento de até 360 mil $\mathrm{t}$ de produção e também haveria a redução da sazonalidade do fruto (PORTAL BRASIL, 2016).

Mas para atingir este objeto é necessário que o melhoramento genético disponha de variabilidade e divergência genética que possibilitem obter as informações necessárias para subsidiar o processo de seleção (Gomes Júnior et al., 2014). Trabalhos 
publicados demonstram a que existe a possibilidade de selecionar genótipos melhorados em progênies de açaizeiros, pois há a presença de plantas se destacando (Yokomizo et al., 2016a; 2016b; Farias Neto et al., 2018).

Um complicador para esta seleção é o efeito que o ambiente tem sobre o comportamento de um material genéticas, cujas variações nas características vegetativas e reprodutivas contribuem de forma diferenciada para o fenótipo final da planta, devido a resposta aos fatores ambientais, fenômeno conhecido como interação genótipos versus ambientes (GxA), que dificulta a seleção e recomendação de genótipos adaptados e estáveis (Cruz et al., 2014). Baseado nestes aspectos, a estatística multivariada possibilita obter informações e interpretações do conjunto de características em estudo, que poderiam não ser perceptíveis com o uso da análise estatística univariada que trabalha com cada uma isoladamente, orientando a tomada de decisões de quais características a serem consideradas, descartandose as redundantes, além disso, os métodos univariados, não consideram a correlação existentes entre as diferentes características (Cruz et al, 2014). Segundo Linden (2009) a análise multivariada resultando em agrupamento tem como objetivo separar objetos diferentes e unir objetos semelhantes, formando grupos distintos com indivíduos similares.

Apesar da importância dos métodos estatísticos multivariados para a interpretação das variações fenotípicas produzidas pelas diferentes características, dá-se preferência a análise univariada em açaizeiros, portanto o objetivo deste trabalho foi analisar o comportamento em conjunto das características avaliadas em progênies desta espécie, pela análise multivariada e componentes principais visando determinar quais atributos contribuem apresentam maior contribuição nas manifestações fenotípicas e também as com maior potencial a serem utilizados em avaliações que se destinem a seleção em gerações futuras.

\section{MATERIAL E MÉTODOS}

Esse estudo é parte do programa de melhoramento genético de açaizeiro para produção de frutos no estuário amazônico, conduzindo duas populações distintas em um plantio de açaizais em área de várzea no Campo Experimental de Mazagão da Embrapa Amapá.

Este Campo Experimental possui solo predominante do tipo Gley Pouco Húmico de textura média, drenado e de média a alta fertilidade natural. A área utilizada apresenta topografia plana e cobertura vegetal tipo capoeira. Apresenta tipo climático Ami segundo classificação de Köppen, tropical chuvoso, com temperatura média do mês mais frio (junho) de $22,7^{\circ} \mathrm{C}$ e do mais quente (setembro) de $32,8^{\circ} \mathrm{C}$, com precipitação do mês mais seco (outubro) de $32 \mathrm{~mm}$ e do mais chuvoso (março) de $365 \mathrm{~mm}$. O regime climático anual apresenta precipitação total de 2410 $\mathrm{mm}$, com concentração entre os meses de janeiro e junho, temperatura média anual de $27^{\circ} \mathrm{C}$ e umidade relativa média do ar de 85\% (CLIMATE-DATA.ORG, 2020).

O material genético utilizado na área é composto por duas populações distintas. A população 1 é originada de sementes coletadas de 75 matrizes selecionadas em açaizais nativos da região oeste da Ilha de Marajó (safra de inverno), a população 2 teve sua origem de 100 matrizes selecionadas na região leste da mesma Ilha (safra na estiagem). O delineamento experimental adotado foi de blocos ao acaso com duas repetições e cada parcela representada por cinco plantas, plantadas no espaçamento de 4 x $5 \mathrm{~m}$. O número reduzido de repetições se deve a quantidade de progênies e a área total do experimento. 
As avaliações foram realizadas mensalmente no período de 2009 a 2013 por meio dos descritores, sendo: FLF - Lançamento Foliar, contagem por planta de folhas em formação, com a bainha foliar fechada; FAB - Folhas Maduras Abertas, contagem de todas as folhas existentes por planta, com exceção das senescidas; FSE - Folhas Senescentes, contagem de todas as folhas senescentes existentes por planta; FC: Número de Facões ou Espatas Emitidas, contagem dos facões ou espatada existentes por planta; FL - Cacho com Inflorescência em Floração, contagem de cachos que apresentam inflorescência em floração por estipe; FV - Formação de Cacho Verde, contagem do número de cachos verdes por estipe; FF- Cachos com Formação de Frutos Verdes, contagem de cachos que apresentam formação de frutos verdes por estipe; FM- Cacho com Frutos Maduros, contagem de cachos que apresentam frutos maduros por estipe; TM - Tamanho do Cacho, valor atribuído a avaliação visual dos cachos: onde 1 é atribuído aos menores valores, e 7 para os maiores; $\mathrm{NE}$ : número de perfilhos presentes em cada estipe e; VS: número de vassouras ou cachos sem frutos.

A análise de componentes principais (ACP) tem como objetivo reduzir a dimensionalidade do conjunto de variáveis e facilitar a interpretação da independência entre elas. Para isso, são obtidas combinações lineares das variáveis originais que geometricamente representam a seleção de novos sistemas de coordenadas, os quais são obtidos pela rotação do sistema original em que há $\mathrm{p}$ variáveis aleatórias como eixos das coordenadas. Esses novos eixos ortogonais (novas variáveis) são chamados de componentes principais e os valores das novas variáveis são chamadas de escores dos componentes principais ou coordenadas principais. Essas novas variáveis não são correlacionadas (Piovesan et al., 2009).

Foram obtidos os componentes principais, de uma forma geral, seja um conjunto de $\mathrm{p}$ variáveis $\mathrm{X}_{1}$,
$\mathrm{X}_{2}, \ldots, \mathrm{X}_{\mathrm{P}}$ com médias $\mu_{1}, \mu_{2}, \ldots, \mu_{\mathrm{P}}$ e variâncias $\sigma^{2}{ }_{1}, \sigma^{2}{ }_{2}$, $\ldots, \sigma^{2} \mathrm{p}$, respectivamente. Estas variáveis não são independentes e, portanto, possuem covariância entre a i-ésima e k-ésima variável definida por $\sigma_{i k}$, para $\mathrm{i} \neq \mathrm{k}, \mathrm{k}$ $=1,2, \ldots$, p. Então as p variáveis podem ser expressas na forma vetorial por: $\mathrm{X}=\left[\mathrm{X}_{1}, \mathrm{X}_{2}, \ldots, \mathrm{X}_{\mathrm{P}}\right]^{\prime}$, com vetor de médias $\mu=\left[\mu_{1}, \mu_{2}, \ldots, \mu_{\mathrm{P}}\right]$ ', e matriz de covariância $\Sigma$. Encontram-se os pares de autovalores e autovetores $\left(\lambda_{1}\right.$, $\left.\mathrm{e}_{1}\right),\left(\lambda_{2}, \mathrm{e}_{2}\right), \ldots,\left(\lambda_{\mathrm{P}}, \mathrm{e}_{\mathrm{P}}\right)$ em que $\lambda_{1} \geq \lambda_{2} \geq \ldots \geq \lambda_{\mathrm{P}}$, associados à $\Sigma$ e, então o i-ésimo componente principal é definido pela seguintes equação: $Z_{i}=e^{\prime}{ }_{i} X=e_{i 1} X_{1}+$ $\mathrm{e}_{\mathrm{i} 2} \mathrm{X}_{2}+\ldots+\mathrm{e}_{\mathrm{ip}} \mathrm{X}_{\mathrm{p}}$, em que $\mathrm{i}=1,2, \ldots, \mathrm{p}$.

A técnica hierárquica de agrupamento consiste em uma série de sucessivos agrupamentos ou sucessivas divisões de elementos, em que os elementos são agregados ou desagregados. Os grupos, na técnica hierárquica, são geralmente representados por um diagrama bi-dimensional chamado de dendrograma ou diagrama de árvore. Nesse diagrama, cada ramo representa um elemento, enquanto a raiz representa o agrupamento de todos os elementos. Para identificação das similaridades do conjunto, foi realizada esta técnica, calculando-se a distância euclidiana para o conjunto das variáveis, e utilizando-se o algoritmo de Ward (1963) para a obtenção dos agrupamentos similares (Freddi et al., 2008). O resultado da análise foi apresentado em um dendrograma, na identificação dos agrupamentos.

A consistência do padrão de agrupamento foi verificada por meio do coeficiente de correlação cofenética (ccc), proposto por Sokal e Rohlf (1962). Este coeficiente é calculado entre os valores de similaridade da matriz original e da matriz resultante da simplificação do dendrograma e mede o grau de ajuste entre a matriz de dissimilaridade (matriz de distância $\mathbf{D}$ ) e a matriz resultante da simplificação devido ao método de agrupamento (matriz cofenética C). Sendo que quanto maior for o valor, menor será a distorção 
provocada pelos agrupamentos dos dados (Vicini e Souza, 2005; Kopp et al. 2007).

Para as análises de dados foi utilizada a linguagem e ambiente do programa $\mathrm{R}$ versão 3.4.1 ( CORE TEAM, 2020). Na execução dessas análises e da técnica de análise de componentes principais foram utilizados os procedimentos princomp e prcomp.

\section{RESULTADOS E DISCUSSÃO}

\section{Análise de Agrupamento}

As combinações de características FC x FSE; FL x FSE; FC x FL; FM x FSE; FC x FM; FL x FM apresentaram os menores valores de medidas de dissimilaridade, neste caso, representam as que mais se assemelharam nas avaliações, podendo-se optar para fins de diminuição em relação ao quantitativo de avaliações por uma destas em relação à outra, por exemplo, com FC x FSE, o melhorista pode escolher trabalhar com FC ou FSE, desta forma pode-se optar após avaliar as combinações do uso apenas de FC e FL. Enquanto que FAB foi a que menos se assemelhou com todas as demais, ao apresentar os valores superiores de dissimilaridade e com isso demonstra conferir aspectos fenológicos muito distinto. As demais combinações apresentaram comportamento intermediário, mas com proximidade às combinações mais semelhantes, enquanto que NE e TM apresentaram pouco maiores, indicando leve dissimilaridade em relação as demais características. Essa dissimilaridade, nas combinações maiores de medidas de dissimilaridade, pode ser utilizada para a escolha dos descritores a serem usados nas avaliações de campo (Tabela 1). Porém, com as avaliações de dissimilaridade somente seriam descartadas as características FM e FSE, mantendo-se nove.

Tabela 1. Medidas de dissimilaridade entre onze caraterísticas ${ }^{1}$ em progênies de açaizeiros com base na distância Euclidiana

\begin{tabular}{|c|c|c|c|c|c|c|c|c|c|c|}
\hline & $\mathrm{NE}$ & FLF & FAB & FSE & $\mathrm{FC}$ & FL & $\mathrm{FF}$ & $\mathrm{FV}$ & FM & TM \\
\hline FLF & 184,36 & & & & & & & & & \\
\hline FAB & 1225,04 & 1331,57 & & & & & & & & \\
\hline FSE & 286,93 & 133,87 & 1458,86 & & & & & & & \\
\hline $\mathrm{FC}$ & 270,25 & 118,45 & 1426,57 & 87,05 & & & & & & \\
\hline FL & 272,23 & 121,22 & 1431,81 & 80,96 & 91,87 & & & & & \\
\hline $\mathrm{FF}$ & 267,32 & 129,84 & 1395,07 & 134,94 & 134,41 & 131,65 & & & & \\
\hline $\mathrm{FV}$ & 271,89 & 128,92 & 1406,80 & 120,27 & 130,35 & 130,27 & 149,17 & & & \\
\hline FM & 281,77 & 128,84 & 1447,01 & 63,57 & 92,27 & 89,11 & 139,99 & 120,90 & & \\
\hline TM & 269,51 & 328,79 & 1039,59 & 449,54 & 420,83 & 425,06 & 395,51 & 406,34 & 442,93 & \\
\hline VS & 280,55 & 137,56 & 1433,65 & 102,40 & 113,55 & 110,99 & 157,41 & 140,98 & 102,66 & 434,56 \\
\hline
\end{tabular}

${ }^{1} \mathrm{NE}$ : número de perfilhos; FLF: lançamento foliar; FAB: folhas maduras abertas; FSE: folhas senescentes; FC: número de espatas; FL: cachos com inflorescência em floração; FF: cachos com formação de frutos verdes; FV: formação de cachos verdes; FM: cachos com frutos maduros; TM: nota do tamanho de cachos; VS: número de vassouras sem frutos.

O ajuste entre a matriz de distâncias e o dendrograma foi estimado pelo coeficiente de correlação cofenética (ccc) (Sokal e Rohlf, 1962), sendo que o ccc acima de 0,7 mostra que o método aqui empregado foi adequado. Caso isso não ocorra, deverse partir para outro método de estimação de distância.
Aqui referente aos dados avaliados. Estimou-se um valor igual a 0,9842, revelando elevado ajuste entre a representação gráfica das distâncias genéticas e a matriz de distância genética original, o que assegura as inferências realizadas por meio da avaliação visual do dendrograma. 


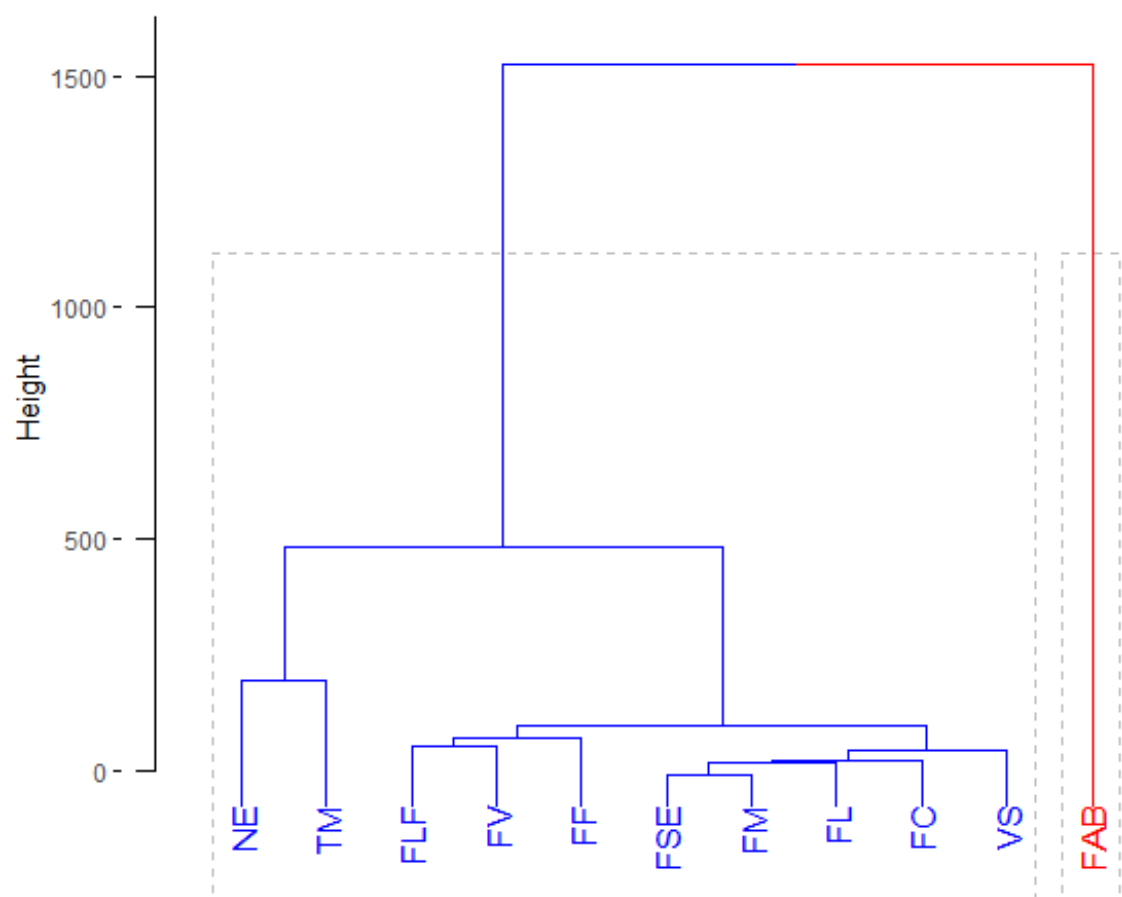

Figura 1. Dendrograma usando matriz de distância euclidiana pelo método Ward em 11 características ${ }^{1}$ fenotípicas em progênies de açaizeiro.

${ }^{1}$ NE: número de perfilhos; FLF: lançamento foliar; FAB: folhas maduras abertas; FSE: folhas senescentes; FC: número de espatas; FL: cachos com inflorescência em floração; FF: cachos com formação de frutos verdes; FV: formação de cachos verdes; FM: cachos com frutos maduros; TM: nota do tamanho de cachos; VS: número de vassouras sem frutos.

A Figura obtida permite a visualização de dois grupos bem distintos entre si, um envolvendo apenas $\mathrm{FAB}$ e o outro com as demais características. Indicando que o grupo contendo as 10 características são similares entre si, não conseguindo distinguir fenotipicamente as progênies e, portanto, para facilitar as avaliações, com menor esforço e tempo despendido pode-se optar em se utilizar uma das características apenas, entre NE,
TM, FLF, FV, FF, FSE, FM, FL, FC e VS, adicionandose FAB. Neste caso avaliações seriam eficientes com apenas duas variáveis em detrimento de 11 , distinto da Tabela 1, onde seriam mantidas nove características. Essa divisão é confirmada pelos resultados observados no método de Elbow (Elbow Method) e o método de silhueta (silhouette). 


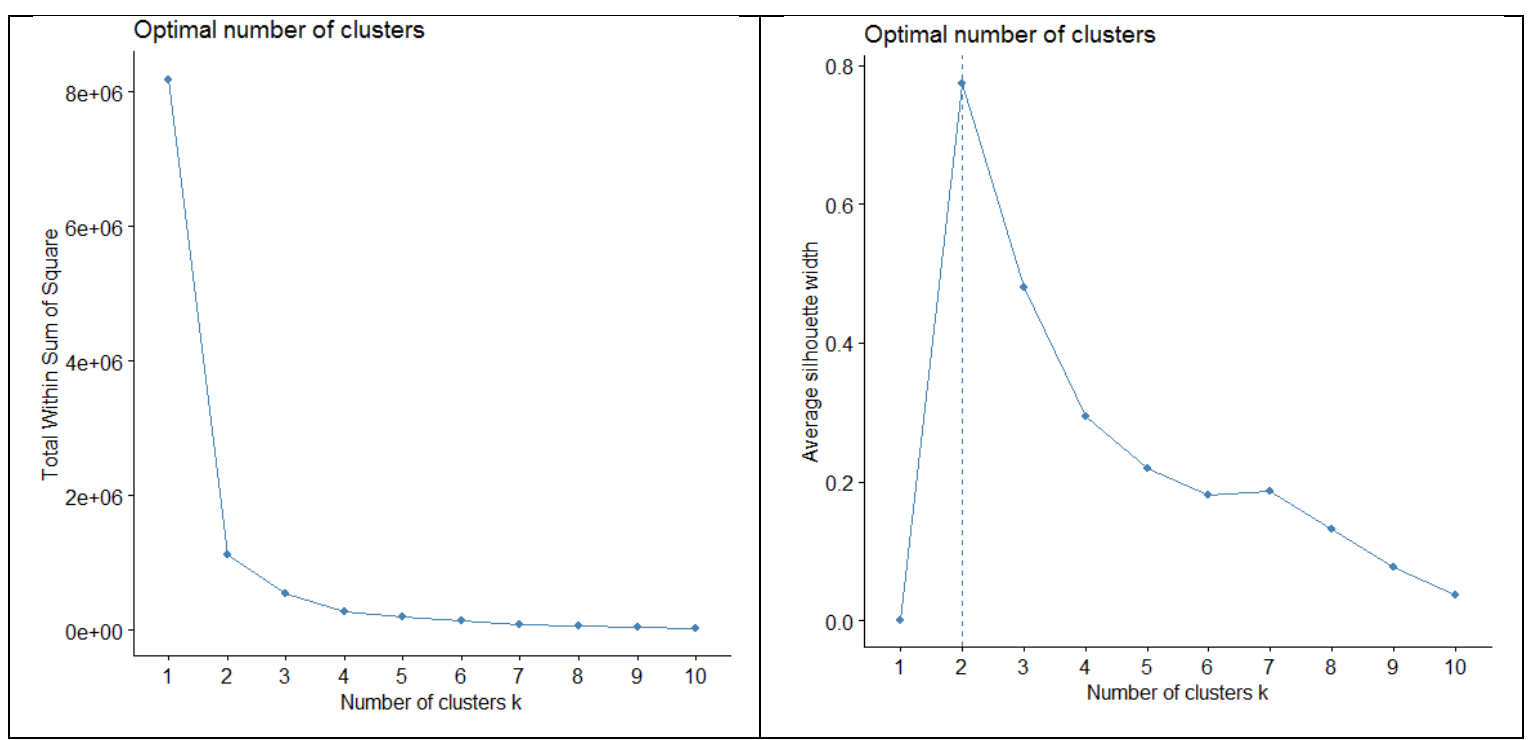

Figura 2. Definição do número ótimo de grupos envolvendo 11 características ${ }^{1}$ em progênies de açaizeiros pelos métodos Elbow (Elbow Method) e da silhueta (Silhouette)

${ }^{1} \mathrm{NE}$ : número de perfilhos; FLF: lançamento foliar; FAB: folhas maduras abertas; FSE: folhas senescentes; FC: número de espatas; FL: cachos com inflorescência em floração; FF: cachos com formação de frutos verdes; FV: formação de cachos verdes; FM: cachos com frutos maduros; TM: nota do tamanho de cachos; VS: número de vassouras sem frutos.

Pelo método de Elbow (Elbow Method) da Figura 2, que analisa a porcentagem de variância entre o número de clusters, identificando num gráfico, a diferença brusca na variância entre os diferentes números de clusters, sinalizando que a adição de mais clusters não interferirá na variância dos dados presentes no conjunto de dados (Kodinariya e Makwana, 2013) e do método de silhueta (silhouette), que combina coesão e divisão, constituindo-se num método de interpretação e validação de consistência dentro dos grupos, onde o valor da silhueta é uma medida de quão similar um objeto é com o seu próprio grupo (coesão) em comparação com outros grupos (separação) segundo
Rousseeuw (1987) e Lee et al. (2012), houve também a indicação que o número ótimo de grupos é igual a dois.

Todavia o uso de apenas duas características para seleção de materiais genéticos pode trazer insegurança ao melhorista em suas avaliações então pode se optar em utilizar três grupos, conforme é mostrado na Figura 3. Tendo-se o primeiro grupo contendo NE e TM, podendo-se optar por uma destas características. No segundo grupo estão presentes FLF, FV, FF, FSE, FM, FL, FC e VS, onde pode-se utilizar uma destas e no terceiro existe apenas uma característica FAB. Conferindo a possibilidade de utilização de três características em avaliações futuras, diminuindo o tempo dispendido e facilitando o processo. 


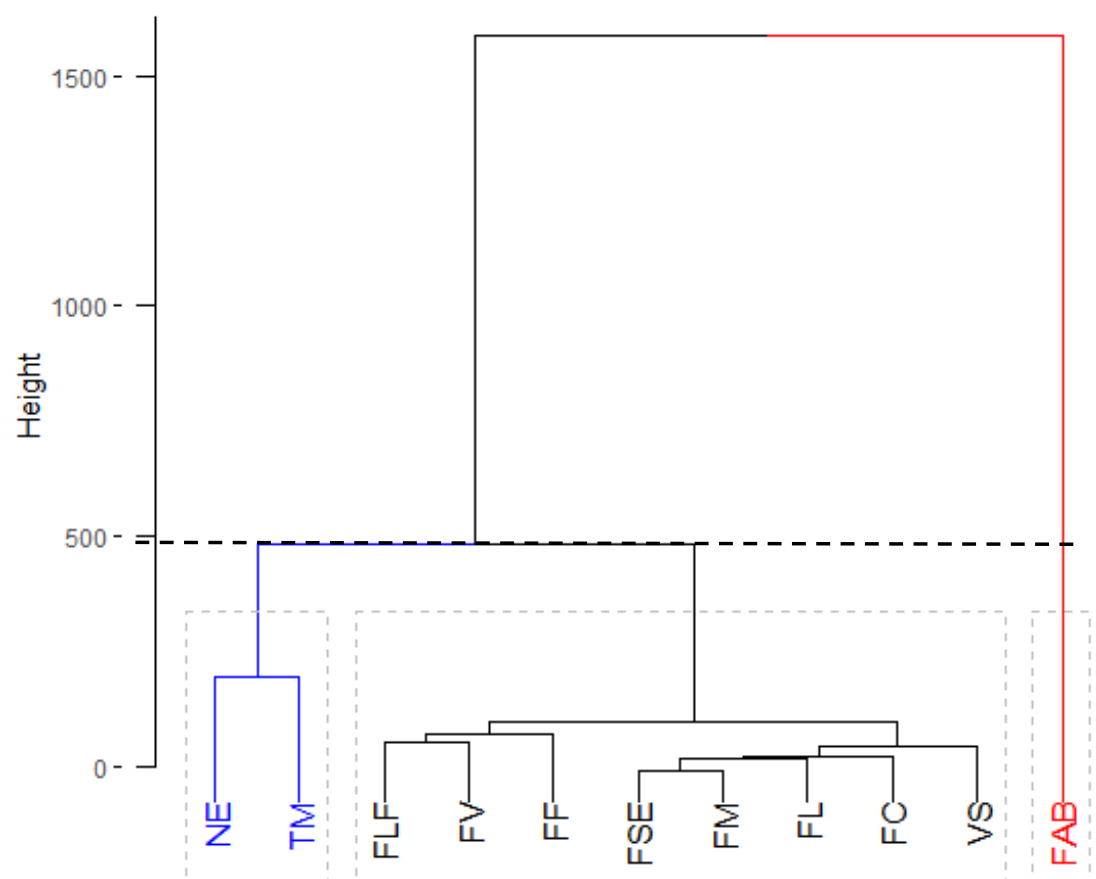

Figura 3. Definição em três grupos envolvendo 11 características $^{1} \mathrm{em}$ progênies de açaizeiros.

${ }^{1} \mathrm{NE}$ : número de perfilhos; FLF: lançamento foliar; FAB: folhas maduras abertas; FSE: folhas senescentes; FC: número de espatas; FL: cachos com inflorescência em floração; FF: cachos com formação de frutos verdes; FV: formação de cachos verdes; FM: cachos com frutos maduros; TM: nota do tamanho de cachos; VS: número de vassouras sem frutos.

\section{Análise de Componentes Principais}

$\mathrm{Na}$ Tabela 2, são apresentadas as variâncias explicadas e cumulativas para cada um dos onze componentes principais, que acumularam e explicaram a totalidade da variabilidade integral dos dados. Não havendo mais componentes apresentando contribuição na variabilidade. Observou-se que os cinco primeiros componentes responderam pela maior parte da variância. Desta forma, não é necessário analisar toda a informação e com isso simplificar a análise, sem a perda de informação. Segundo Andrade et al. (2007), esses fatores comuns e independentes (componente principal) reduzem a dimensão de variáveis interrelacionadas em dimensões menores, para explicar a variabilidade dos dados originais.

$\mathrm{Na}$ análise inter-relacionada todas as características apresentaram função discriminante canônica positiva em pelo menos uma das cinco primeiras variáveis, conforme apresentado na Tabela 3 , indicando que estas características foram responsáveis para as variações observadas nas progênies excetuandose apenas VS. Notando-se que a característica que apresentou maior valor positivo para a variável canônica 1 foi $\mathrm{FAB}$, demonstrando que foi o de maior peso na separação das progênies, seguida por TM, FV e FF. Já as demais características apresentaram um efeito de supressão na separação das progênies avaliadas. Destas, as menos discriminantes foram NE e VS, podendo-se considerá-las como pouco relevantes em estudos de divergência genética e identificação de duplicidade nas progênies avaliadas. 
Tabela 2. Autovalores da análise dos componentes principais, variâncias explicadas percentuais e variância acumulada, visando estimar a dissimilaridade entre 11 características $^{1} \mathrm{em}$ açaízeiros

\begin{tabular}{cccc}
\hline & Autovalor & $\mathrm{V}^{2 \%}$ & $\mathrm{~V}^{2 \%}$ ocum \\
\hline CP1 & 1,9673 & 32,6896 & 32,6896 \\
CP2 & 0,9057 & 15,0486 & 47,7382 \\
CP3 & 0,8851 & 14,7070 & 62,4452 \\
CP4 & 0,6623 & 11,0044 & 73,4496 \\
CP5 & 0,5116 & 8,5002 & 81,9498 \\
CP6 & 0,4243 & 7,0501 & 88,9999 \\
CP7 & 0,2434 & 4,0450 & 93,0449 \\
CP8 & 0,2081 & 3,4582 & 96,5031 \\
CP9 & 0,1306 & 2,1709 & 98,6740 \\
CP10 & 0,0570 & 0,9473 & 99,6213 \\
CP11 & 0,0228 & 0,3787 & 100,0000 \\
\hline
\end{tabular}

${ }^{1} \mathrm{NE}$ : número de perfilhos; FLF: lançamento foliar; FAB: folhas maduras abertas; FSE: folhas senescentes; FC: número de espatas; FL: cachos com inflorescência em floração; FF: cachos com formação de frutos verdes; FV: formação de cachos verdes; FM: cachos com frutos maduros; TM: nota do tamanho de cachos; VS: número de vassouras sem frutos.

Tabela 3. Escores canônicos padronizados para as cinco primeiras funções discriminantes canônicas (FDCs) entre as 11 características originais

\begin{tabular}{cccccc}
\hline & CP1 & CP2 & CP3 & CP4 & CP5 \\
\hline NE & $-0,6866$ & 0,5476 & 0,3943 & 0,1200 & 0,1753 \\
FLF & 0,0578 & $-0,0296$ & $-0,0516$ & 0,0164 & 0,0348 \\
FAB & $\mathbf{0 , 8 2 6 2}$ & 0,0498 & 0,5600 & 0,0036 & $-0,0172$ \\
FSE & 0,0561 & $-0,0441$ & $-0,0625$ & $-0,0195$ & 0,0860 \\
FC & 0,0966 & $-0,1683$ & $-0,1624$ & 0,0450 & $-0,2093$ \\
FL & 0,0704 & $-0,1152$ & $-0,1747$ & 0,1029 & $-0,1679$ \\
FF & 0,4127 & $-0,0121$ & $-0,2724$ & 0,8226 & 0,2274 \\
FV & 0,4377 & 0,1102 & $-0,2724$ & $-0,3899$ & 0,7478 \\
FM & 0,0654 & $-0,2288$ & 0,0163 & $-0,1584$ & 0,1155 \\
TM & 0,4906 & 0,7165 & $-0,4008$ & $-0,1162$ & $-0,2514$ \\
VS & $-0,0010$ & $-0,3365$ & $-0,0252$ & $-0,2688$ & $-0,0548$
\end{tabular}

${ }^{1} \mathrm{NE}$ : número de perfilhos; FLF: lançamento foliar; FAB: folhas maduras abertas; FSE: folhas senescentes; FC: número de espatas; FL: cachos com inflorescência em floração; FF: cachos com formação de frutos verdes; FV: formação de cachos verdes; FM: cachos com frutos maduros; TM: nota do tamanho de cachos; VS: número de vassouras sem frutos.

As características FSE, FLF e FL apesar de características geradas pelas progênies. Podendo ser serem importantes na diferenciação das progênies, importantes em populações mais homogêneas. tiveram baixo fator discriminante, o que pode ser explicado pela baixa heterogeneidade nestas 


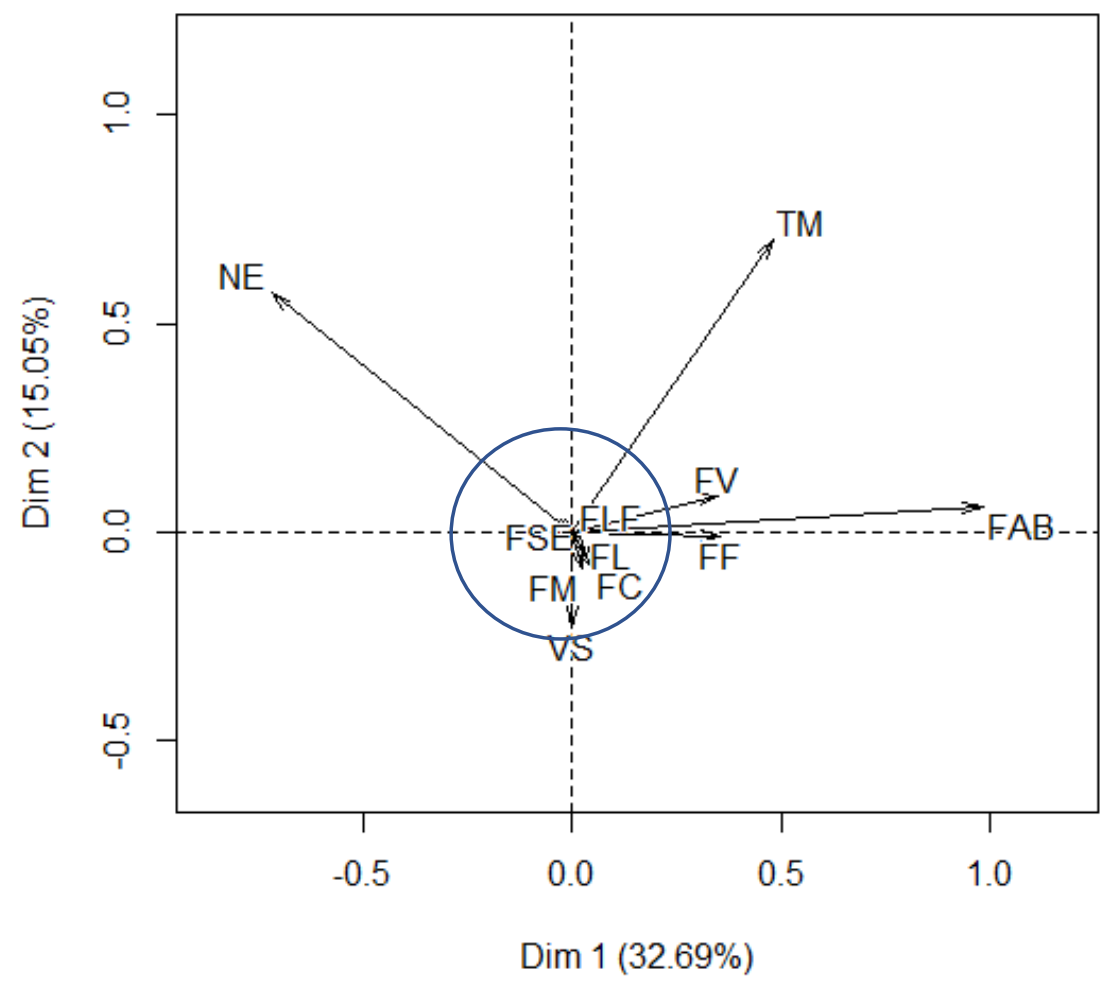

Figura 4. Dispersão gráfica dos escores dos dois primeiros componentes principais para 11 características $^{1}$ fenotípicas em progênies de açaizeiro.

Dispersão gráfica dos escores dos dois primeiros componentes principais $\mathrm{p}$

${ }^{1} \mathrm{NE}$ : número de perfilhos; FLF: lançamento foliar; FAB: folhas maduras abertas; FSE: folhas senescentes; FC: número de espatas; FL: cachos com inflorescência em floração; FF: cachos com formação de frutos verdes; FV: formação de cachos verdes; FM: cachos com frutos maduros; TM: nota do tamanho de cachos; VS: número de vassouras sem frutos.

Além dos dados estatísticos, é importante ressaltar que uma das faculdades mais bem desenvolvidas e agudas do cérebro humano é a capacidade de perceber, analisar e interpretar informações visuais complexas, como os existentes em gráficos. Padrões, ou relações visuais, são os mais fáceis de entender rapidamente, enquanto que dados tabulados precisam ser mais detalhadamente processados. A compreensão da informação visual é muito melhor e mais rápida que a informação numérica linear ou tabular, conforme afirma citação em Yan e Kang (2003), com isso o GGE biplot torna-se uma importante ferramenta auxiliar ao melhorista e foi realizado visando complementar e ratificar os resultados obtidos numéricamente.

A inter-relação entre as características nas progênies de açaizeiro é demonstrada pela proximidade entre os ângulos de suas setas. Já a importância destas variáveis em explicar ou contribuir para a variabilidade é visualizada pelo comprimento das setas (Melém et al., 2008).

Quando as características estão em setores distintos do Biplot, há indicativos que existe interrelação contrária ente si e, quando presentes nos mesmos setores há inter-relação positiva. Com base nas relações, TM é independente e NE está negativamente correlacionado em relação ao grupo FAB, FV e FF, e estas três estão inter-relacionadas de forma positiva entre si. Visualmente FC, FLF, FL, FM, FSE e VS entre si e apresentam inter-relação negativa com TM e o primeiro grupo de forma praticamente independente de FAB, FV e FF.

Quanto as características redundantes, ou seja, aquelas que podem ser descartadas nas avaliações de 
campo, que tem como reflexo o dispêndio adicional de recursos econômicos e humanos, além de tempo do melhorista, no Biplot, são aquelas que as retas estão muito próximas entre si. Adicionalmente, de forma semelhante nas redundantes, pode-se dispensar aquelas que contribuem muito pouco com a variabilidade.

A característica FAB apresentou o maior peso contribuindo mais intensamente de forma positiva para o primeiro componente principal, seguido por TM, enquanto que FC, FLF, FL, FM, FSE e VS, praticamente não contribuíram para a variabilidade capturada no primeiro eixo componente. As características FF e FV também contribuíram para a variabilidade captada no primeiro eixo, mas apresentaram as menores contribuições. No segundo eixo componente destacaram-se NE e TM. De forma simplória o melhorista poderia optar por avaliar FAB, TM, e também FF e FV e descartar todo o grupo FC, FLF, FL, FM, FSE e VS, que apresentou pouca contribuição nos dois primeiros componentes principais, com isso ter-se-ia apenas quatro características a serem avaliadas em comparação as 11 iniciais. De modo geral, apesar das diferenças entre 11 as características, observa-se que a maioria se concentra próximas no centro da Figura 4, mostrando-se menos divergentes entre si, ou seja, apresentam aparente redundância. Esta proximidade entre estas características especificamente pode indicar baixa variabilidade entre as progênies. Entretanto, nota-se três características (NE, TM e FAB) dispersas das que se encontram concentrados no centro da Figura 4. Essa dispersão confirma a maior capacidade de diferenciação das progênies nestas características.

\section{CONCLUSÕES}

A análise inicial de Elbow e Silhueta indicam para seleção o uso de FAB e outra dentre as demais, semelhante ao dendrograma;
Para seleção pelo dendrograma pode-se utilizar FAB, escolher entre NE ou TM e na terceira característica optar entre as demais restantes;

O Biplot refinou a escolha das caraterísticas, permitindo visualizar que FAB, TM, FV e FF, com contribuições positivas devem ser utilizadas para seleção;

O uso de metodologias combinadas, principalmente com o Biplot, permite corrigir a escolha das características prioritárias dentro de uma ampla quantidade.

\section{REFERÊNCIAS}

ANDRADE, E.M.; ARAÚJO, L.F.P.; ROSA, M.F; GOMES, R.B.; LOBATO, F.A. Fatores determinantes da qualidade das águas superficiais na bacia do Alto Acaraú, Ceará, Brasil. Ciência Rural, v.37, n.6, p.1791-1797, 2007.

CARVALHO, A.V.; SILVEIRA, T.F.F.; MATTIETTO, R.A.; OLIVEIRA, M.S.P.; GODOY, H.T. Chemical composition and antioxidante capacity of açaí (Euterpe oleracea) genotypes and commercial pulps. Journal of the Science of Food and Agriculture, v.97, p.1467-1474, 2017. DOI: 10.1002/jsfa.7886.

CLIMATE-DATA.ORG Clima Mazagão. Disponível em: <https://pt.climate-data.org/america-dosul/brasil/amapa/mazagao-33758/\#climategraph>. Acesso em: 11 mai 2020.

COMEX DO BRASIL. No Pará, produção de açaí deve ter mais assistência técnica e investimentos, informa o Mapa. 2017. Disponível em: <https://www.comexdobrasil.com/no-paraproducao-de-acai-deve-ter-maisassistenciatecnica-e-investimentos-informa-Omapa/>. Acesso em: 11 mai 2020.

CRUZ, C.D.; CARNEIRO, P.C.S.; REGAZZI, A.J. Modelos biométricos aplicados ao melhoramento genético (3. ed.). Viçosa: Editora UFV. 2014. 668 p.

FARIAS NETO, J. T. de; YOKOMIZO, G. K. I.; OLIVEIRA, M. do S. P. de; HONGYU, K. GGE Biplot para estabilidade e adaptabilidade em progênies de açaizeiro de Anajás, PA. 
Revista Agro@mbiente On-line, v. 12, n. 1, p. 409-417, 2018.

FARIAS NETO, J.T.; RESENDE, M.D.V.; OLIVEIRA, M.S.P. Seleção simultânea em progênies de açaizeiro irrigado para produção e peso do fruto. Revista Brasileira de Fruticultura, v.33, n.1, p.532-539, 2011. doi.org/10.1590/S0100-29452011000200025.

FREDDI, O. S.; FERRAUDO, A. S.; CENTURION, J. F. Análise multivariada na compactação de um Latossolo Vermelho cultivado com milho. Revista Brasileira de Ciência do Solo, v.32, p. 953-961, 2008.

GOMES JÚNIOR, G.M. Abordagem genética e multivariada na performance agronômica de genótipos de soja oriundos de diferentes genealogias. 2014, 79p. (Doutorado Universidade Estadual Paulista, Faculdade de Ciências Agrárias e Veterinárias), 2014.

IBGE Produção Agrícola Municipal - PAM, 2018. Disponível em:

$<$ https://sidra.ibge.gov.br/tabela/1613>.

Acesso em: 13 abr. 2020

KODINARIYA, T.M.; R. MAKWANA, P.R Review on determining number of Cluster in K-Means Clustering. International Journal of Advance Research in Computer Science and Management Studies, v.1, n.6, p.90-95, 2013

KOPP, M.M.; DE SOUZA, V.Q.; COIMBRA, J.L.M.; LUZ, V.K.; MARINI, N.; OLIVEIRA, A.C. Melhoria da correlação cofenética pela exclusão de unidades experimentais na construção de dendrogramas. Revista da FZVA, v.14, n.2, p.46-53, 2007

LEE, K.; LEE, K.; LEE, C. Statistical cluster validity indexes to consider cohesion and separation. International conference on Fuzzy Theory and Its Applications (iFUZZY2012), p.p. 228-232, 2012.

LINDEN, R. Técnicas de Agrupamento. Revista de Sistemas de Informação da FSMA, n.4, p.1836, 2009.

MELÉM JÚNIOR, N.J.; FONSECA, I.C.B.; BRITO, O.R.; DECAËNS, T.; CARNEIRO, M.M.; MATOS, M.F.A.; GUEDES, M.C.; QUEIROZ, J.A.L.; BARROSO, K.O. Análise de componentes principais para avaliação de resultados analíticos da fertilidade de solos do Amapá. Semina: Ciências Agrárias, v.29, p.499-506, 2008.

MENEZES, E.M.S. Efeito da alta pressão hidrostática em polpa de açaí pré-congelada (Euterpe oleracea, Mart.). Dissertação de Mestrado (Ciência e Tecnologia de Alimentos), Universidade Federal Rural do Rio de Janeiro, Seropédica, RJ. 2005; 83p.

MOURA ROCHA, S. M. B. De. Benefícios funcionais do açaí na prevenção de doenças cardiovasculares. Journal of Amazon Health Science, v.1, n. 1, p. 1-10, 2015.

NEVES, L.T.B.C.; CAMPOS, D.C.S.; MENDES, J.K.S.; URNHANI, C.O.; ARAÚJO, K.G.M. Quality of fruits manually processed of açaí (Euterpe oleracea Mart.) and bacaba (Oenocarpus bacaba Mart.). Revista Brasileira de Fruticultura, v. 37, n. 3, p. 729-738, 2015.

NOGUEIRA, A.K.M.; SANTANA, S.C. de; GARCIA, W.S. A dinâmica do mercado de açaí fruto no Estado do Pará: de 1994 a 2009. Revista Ceres, v. 60, n.3, p. 324-331, 2013.

PAGLIARUSSI, M.S. A cadeia produtiva agroindustrial do açaí: estudo da cadeia e proposta de um modelo matemático. Monografia. Universidade de São Paulo. São Carlos - SP: 2010; 66p.

PESSOA, J.D.C.; TEIXEIRA, G. H. de A. Tecnologias para inovação nas cadeias euterpe. Brasília, DF: Embrapa, 2012. 343p.

PIOVESAN, P.; ARAÚJO, L. B.; DIAS, C.T.S. Validação cruzada com correção de autovalores e regressão isotônica nos modelos de efeitos principais aditivos e interação multiplicativa. Ciência Rural, v.39, p.1018-1023, 2009.

PORTAL BRASIL, 2016. Pará ganha programa para ampliar produção de açaí. Disponível em: $<$ http://www.brasil.gov.br/ciencia-etecnologia/2016/01/para-ganhaprograma-paraampliar-producao-de-acai $>$. Acesso em: 13 abr. 2016.

R CORE TEAM. R: A language and environment for statistical computing. R Foundation for Statistical Computing, Vienna, Austria. 2020. 
Disponível em:<http://www.R-project.org/. $\geq$. Acesso em: 20 abr 2020.

RODRIGUES, E. C. N.; RIBEIRO, S. da C.; SILVA, F. L. da. Influência da cadeia produtiva do açaí (Euterpe oleracea Mart.) na geração de renda e fortalecimento de unidades familiares de produção, Tomé Açu-PA. Obeservatorio de la economía Latinoamericana, n. 210, 2015a.

RODRIGUES, P. L.; ALVES FILHOS, P.P.C.; GOMES, A.A.C.; SILVA, J.L. da; LIMA, D.A. Atividades desenvolvidas pelas famílias no extrativismo do açaí (Euterpe oleracea Mart.) em Ponta de Pedras-PA. 2015, CONTECC, Fortaleza, CE, p.4, 2015b.

ROUSSEEUW, P.J. Silhouettes: A graphical aid to the interpretation and validation of cluster analysis. Journal of Computational and Applied Mathematics, v. 20, p.p. 53-65, 1987.

SILVA, A.C.D.; SMIDERLE, O.J.; OLIVEIRA, J.M.F.; SILVA, T.J. Tamanho da semente e substratos na produção de mudas de açaí. Advances in Forestry Science, v. 4, n. 4, p. 51-156, 2017.

SILVESTRE, W.V.D.; PINHEIRO, H.A.; SOUZA, R.O.R.M.; PALHETA, L.F. Morphological and physiological responses of açaí seedlings subjected to diferente watering regimes. Revista Brasileira de Engenharia Agrícola e Ambiental, v.20, n.4, p.364-371, 2016.

SOKAL, R.R.; ROHLF, F.J. The comparison of dendrograms by objective methods. Taxon, v.11, p.30-40, 1962.

SOUZA, M.O.; SANTOS, R.C.; SILVA, M.E.; PEDROSA, M.L. Açaí (Euterpe oleraceae Martius): chemical composition and bioactivity. Nutrire: Revista Sociedade Brasileira de
Alimentação e Nutrição, v.36, n.2, p.161- 169, 2011.

TAVARES, G. dos S.; HOMMA, A. K. O. Comercialização do açaí no Estado do Pará: Alguns comentários. Revista Observatorio de la Economia Latino americana, Brasil, n. 211, 2015. Disponivel em: <https://www.embrapa.br/amazoniaoriental/busca-de-publicacoes//publicacao/1031486/comercializacao-do-acaino-estado-do-para-alguns-comentarios $>$

VICINI, L; SOUZA, A. M. Análise multivariada da teoria à prática. Santa Maria: UFSM; CCNE, 2005. 215 p.

YAMAGUCHI, K.K.L.; PEREIRA, L.F.R.; LAMARÃO, C.V.; LIMA, E.S.; VEIGAJÚNIOR, V.F. Amazon acai: Chemistry and biological activities: A review. Food chemistry, v. 179, p. 137-151, 2015.

YAN, W.; KANG, M.S. GGE biplot analysis: A graphical tool for breeders, geneticists, and agronomists. CRC Press, BocaRaton, FL, 2003, 271p.

YOKOMIZO, G. K. I.; FARIAS NETO, J. T. de; OLIVEIRA, M. do S. P. de Ganho esperado na seleção de progênies de polinização aberta de Euterpe oleracea para produção de frutos. Scientia Forestalis, Piracicaba, v. 44, n. 109, p. 241-248, 2016a.

YOKOMIZO, G. K.-I.; MOCHIUT'TI, S.; QUEIROZ, J. A. L. de; SANTOS, G. R. dos; FURTADO, R. G.; BRANDÃO, A. P.; COLARES, I. B. Estimativas de parâmetros genéticos para caracteres de frutos em açaizeiros no Amapá. Ciência Florestal, Santa Maria, v. 26, n. 3, p. 985-993, 2016b. 